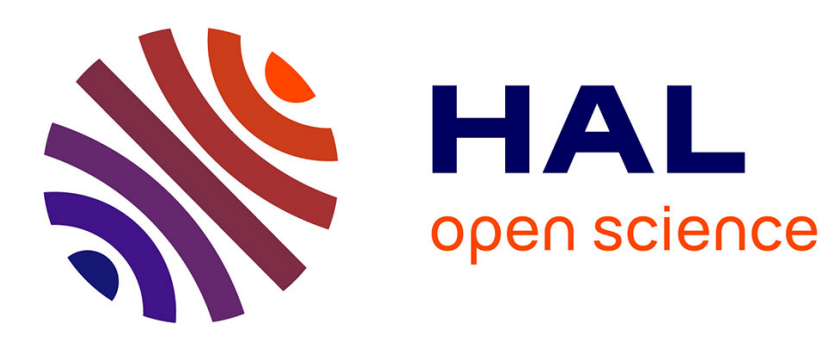

\title{
Simplified variational and physical analysis of heat transfer in laminar and turbulent flow
}

Maurice A. Biot

\section{To cite this version:}

Maurice A. Biot. Simplified variational and physical analysis of heat transfer in laminar and turbulent flow. Physics of Fluids, 1967, 10 (7), pp.1424-1437. 10.1063/1.1762302 . hal-01368608

\section{HAL Id: hal-01368608 \\ https://hal.science/hal-01368608}

Submitted on 21 Sep 2016

HAL is a multi-disciplinary open access archive for the deposit and dissemination of scientific research documents, whether they are published or not. The documents may come from teaching and research institutions in France or abroad, or from public or private research centers.
L'archive ouverte pluridisciplinaire HAL, est destinée au dépôt et à la diffusion de documents scientifiques de niveau recherche, publiés ou non, émanant des établissements d'enseignement et de recherche français ou étrangers, des laboratoires publics ou privés. 


\section{AIP $\left.\right|_{\text {Physics of }}$ Fluids}

Simplified Variational and Physical Analysis of Heat Transfer in Laminar and Turbulent Flow

M. A. Biot

Citation: Physics of Fluids 10, 1424 (1967); doi: 10.1063/1.1762302

View online: http://dx.doi.org/10.1063/1.1762302

View Table of Contents: http://scitation.aip.org/content/aip/journal/pof1/10/7?ver=pdfcov

Published by the AIP Publishing

\section{Articles you may be interested in}

Comparison of Different Correlating Methods for the Single-Phase Heat Transfer Data in Laminar and Turbulent Flow Regions

AIP Conf. Proc. 1233, 614 (2010); 10.1063/1.3452245

Convective heat transfer in unsteady laminar parallel flows

Phys. Fluids 18, 103602 (2006); 10.1063/1.2359742

Augmentation of heat and mass transfer in laminar flow of suspensions: A correlation of data J. Appl. Phys. 51, 791 (1980); 10.1063/1.327293

HEAT TRANSFER TO LIQUID HELIUM IN NARROW CHANNELS WITH LAMINAR AND TURBULENT FLOW

Appl. Phys. Lett. 17, 125 (1970); 10.1063/1.1653332

Effects of Turbulence on Laminar Skin Friction and Heat Transfer

Phys. Fluids 9, 2337 (1966); 10.1063/1.1761623

\section{Looking for a specific} instrument?

Easy access to the latest equipment.

Shop the Physics Today Buyer's Guide.

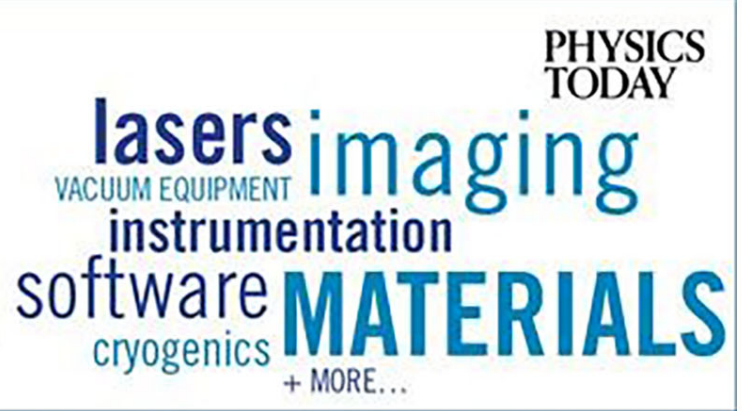




\title{
Simplified Variational and Physical Analysis of Heat Transfer in Laminar and Turbulent Flow
}

\author{
M. A. Biot \\ New York, New York \\ (Received 16 May 1966; final manuscript received 9 March 1967)
}

\begin{abstract}
The fundamental physical features of convective heat transfer for laminar and turbulent flow in ducts and boundary layers are brought out and analyzed quantitatively. A simplified variational method is used which is shown to be remarkably accurate by applying it to special cases where exact solutions are known. Results are expressed in nondimensional form by means of two universal trailing functions which embody the essential features of heat transfer for laminar and turbulent flow and are approximated by very simple piece-wise analytic expressions. The results are applicable to nonparallel streamlines as encountered in duct entry and most boundary layers. This is achieved by introducing a new transformation which provides a suitable extension of the "conduction analogy" to these cases. Drastic simplifications are derived in the analysis of convective heat transfer. In particular it is shown how the trailing function is used in evaluating the heat transfer in heat exchangers and in the much more complex problem of transient heat conduction in a structure adjacent to a moving fluid. In the latter case it is pointed out how the use of the trailing function avoids serious errors in thermal stress analysis.
\end{abstract}

\section{INTRODUCTION}

$\mathrm{A}$ $\mathrm{N}$ important branch of the physics of fluids is concerned with mass and energy transport due to the combined effect of diffusion and convection.

The field equations which govern these phenomena have long been known and a large collection of analytical and numerical solutions are available in the literature. However, the formalistic and numerical manipulations of the fundamental equations are not sufficient in order to provide a really comprehensive theory from the standpoint of the physicist. The problem still remains to construct a physical model which brings to light the significant factors and the corresponding dimensionless parameters along with sound quantitative justification for the exclusion of many others which are not fundamentally relevant to the physics. In addition to new physical insight, this viewpoint leads to the clarifcation of a number of paradoxical results as well as much needed simplification and an intuitive understanding.

In particular this approach provides a simple and physically correct evaluation of the author's trailing function $^{1}$ which was introduced to represent completely the convective heat transfer properties as a practical tool in technological applications thereby eliminating the use of the inadequate concept of "local" heat transfer coefficient. Once the trailing function is known it is possible to formulate correctly and in a simple way a large class of thermal problems involving heat transfer and transient temperatures.

${ }^{1}$ M. A. Biot, J. Aerospace Sci. 29, 568 (1962).
As regards the applications, one of the crucial problems of aerospace technology is the evaluation of transient temperatures and the associated thermal stresses in a flight structure bounded by a moving fluid. Because of the extreme complexity of the phenomena involved current procedures of analysis make use of the simplifying concept of local heat transfer coefficient. However, that this concept is fundamentally wrong is shown by a detailed analysis of the physical nature of heat transfer in a moving fluid. ${ }^{2}$ Results of this analysis also indicate that the use of the local heat transfer coefficient may lead to serious errors particularly in the evaluation of thermal stresses, since they do not depend on the magnitude of the temperatures but on their differences. Such conditions occur in complex flight structures. For example at junctions of webs and flanges local aerodynamic heating and cooling generate transient differential temperatures which are not localized and remain appreciable at relatively large distances downstream. Such effects are essentially convective and cannot be accounted for by local heat transfer properties.

The variational Lagrangian procedures introduced by the writer ${ }^{1}$ avoid these difficulties and provide a physically correct and relatively simple formulation of transient thermal conduction coupled to convective heat transfer at the boundary.

A key to the variational formulation is the concept of trailing function as defined earlier by the author in a very general context. ${ }^{1}$ As illustrated in the last paragraph of this paper the trailing function also

\footnotetext{
${ }^{2}$ M. A. Biot, J. Aerospace Sei. 29, 558 (1962).
} 
provides at the same time convenient solutions of simple problems such as those of forced convection and heat exchangers.

The trailing function itself is easily evaluated by variational procedures with remarkable accuracy. As shown previously ${ }^{1}$ by simple examples, this is accomplished by using a conduction analogy. By this analogy the convection problem is replaced by a mathematically equivalent one of pure conduction which is then solved by the variational methods developed earlier for pure conduction. ${ }^{3}$

The purpose of the present paper is to provide a physical and intuitive analysis of convective heat transfer using variational methods and to derive typical trailing functions for laminar and turbulent flow. An important result also derived below, is a new conduction analogy for the case of nonparallel streamlines. As previously suggested, the von Mises transformation $^{2}$ provides a conduction analogy for this case. However, the resulting analogy is physically awkward and in the application of variational methods leads to difficulties which are avoided by the new equation derived in Sec. VIII below.

The analysis has been focused on bringing to light fundamental and essential features. By a suitable choice of key parameters we show that the trailing function is not very sensitive to the velocity profile and may be represented approximately by only two universal piece-wise analytic functions, one for laminar and one for turbulent flow. By a simple procedure these functions are applicable to boundary layers with nonparallel streamlines. As a consequence they also provide practical methods of analyzing heat transfer in ducts under entrance conditions.

The two basic trailing functions must be considered as first approximations which should be satisfactory in many types of practical problems. Refinements may of course be introduced leading to small corrections as exemplified in Sec. VI. Such refinements and corrections should also be the object of further investigations for velocity profiles which differ drastically from the more general types assumed in the present paper.

Finally it should be noted that the simplicity of the method as applied to problems of convective heat transfer with a known velocity field of the fluid suggests the possibility of solving without the need of large computers the much more complex case where the fluid velocity is unknown and depends on the heat transfer itself.

${ }^{3}$ M. A. Biot, J. Aeron. Sci. 24, 857 (1957).
There exists extensive literature on boundary layer heat transfer, much of it in the nature of problem-solving using methods developed in the fundamental papers of Leveque ${ }^{4}$ and Lighthill. ${ }^{5}$ The present paper constitutes an attempt to use the author's recently developed variational methods to provide new physical insight as well as drastic simplification in the analysis of convertive heat transfer.

\section{TRAILING FUNCTION AND THE CONDUCTION ANALOGY}

In its general form the trailing function is defined as follows. Consider that heat is injected into the fluid at a point $P^{\prime}$ of the boundary at a constant rate per unit time. This causes an increase of temperature of the fluid at the boundary. This increase in temperature at any other point $P$ of the boundary is $r\left(P, P^{\prime}\right)$. In the absence of heat transfer from the solid, the boundary temperature is the adiabatic temperature $\theta_{a}(P)$. The function $r\left(P, P^{\prime}\right)$ defines the trailing function for the injection point. Note that $r$ is singular and infinite for $P=P^{\prime}$ since a finite rate of heat flow is concentrated at the point of injection. Strictly speaking for a time-dependent heat flow the trailing function should be also a function of time. However as a quasi-steady approximation the steady-state expression of the trailing function may be used as if the heat injection rate and the fluid flow were time-independent. The range of validity of this approximation was discussed earlier. ${ }^{2}$

In general, and for three-dimensional flow the trailing function $r\left(P, P^{\prime}\right)$ is defined on a surface. In many applications the flow is two-dimensional in the $x, y$ plane. In such a case it is convenient to modify slightly the definition of the trailing functions as follows. Assume the case of a straight boundary along the $x$ axis. Injection into the fluid is concentrated on a line at the boundary instead of at a point. The line is perpendicular to the $x, y$ plane and the heat injected per unit length of this line and for unit time is equal to unity. If the injection line if located at the abscissa $\xi$, the temperature rise at a point $x$ of the boundary is expressed in the form

$$
\theta-\theta_{a}=r(x-\xi, \xi) .
$$

Note that this function corresponds to concentrated injection of heat per unit length on a line. Hence its physical dimension is not the same as for the

\footnotetext{
A. Leveque, Ann. Mines 13, 201, 381 (1928). (1950)
} 
case where injection is concentrated at a point.

In the following we consider a slab of fluid of unit thickness parallel to the $x, y$ plane and we speak of a concentrated injection of heat at point $x=\xi$ of the boundary. It is understood that this refers to heat injection per unit length and concentrated on a line located at point $x=\xi$ and perpendicular to the $x, y$ plane.

Evaluation of the trailing function may be carried out by using a conduction analogy as already outlined earlier. ${ }^{2}$ For example consider a two-dimensional laminar flow with parallel streamlines. The $x$ axis lies along the plane boundary and the fluid occupies the region $y>0$. The velocity profile of the boundary layer is

$$
u=u(y) .
$$

In the linearized problem, we may put $\theta_{a}=0$ without loss of generality. Hence the excess temperature $\theta$ above the adiabatic value satisfies the equation

$$
c u(\partial \theta / \partial x)=k\left[\left(\partial^{2} \theta / \partial x^{2}\right)+\left(\partial^{2} \theta / \partial y^{2}\right)\right] .
$$

The fluid is assumed to be incompressible, of heat capacity $c$ per unit volume and thermal conductivity $k$, both of constant value.

It has long been known that the term $\partial^{2} \theta / \partial x^{2}$ in Eq. (3.3) may be neglected in a large majority of problems. This approximation was already used by Leveque $^{4}$ in his classical paper. The validity of the approximation was also discussed more recently. ${ }^{2}$ This leads to the simplified equation

$$
c u(\partial \theta / \partial x)=k\left(\partial^{2} \theta / \partial y^{2}\right) .
$$

We may identify $x$ with a time variable $t$ and

$$
c^{\prime}(y)=c u,
$$

with a heat capacity function of $y$. Equation (2.4) becomes

$$
c^{\prime}(y)(\partial \theta / \partial t)=k\left(\partial^{2} \theta / \partial y^{2}\right) .
$$

This equation coincides with that of a transient heat conduction problem where a semi-infinite solid of thermal conductivity $k$ replaces the fluid, and the heat capacity $c^{\prime}(y)$ of the solid depends on the distance $y$ from the boundary. Hence the temperature field $\theta$ is obtained by evaluating the penetration of heat by conduction in the solid, thus we are led to an analog model for the convective problem. We have referred to this as a conduction analogy. ${ }^{2}$ This analogy is much more general than the particular example represented by Eq. (2.6).

The analogy may be used to evaluate the trailing function. Mathematically the problem may be formulated as follows.

Consider Eq. (2.4) and let both sides be integrated from $y=0$ to $y=\infty$. We derive

$$
\int_{0}^{\infty} c u(\partial \theta / \partial x) d y=-k(\partial \theta / \partial y)_{y=0} .
$$

Note that the right side of this equation represents the rate of heat injection per unit time and unit area into the fluid at the boundary. If the total rate of heat injection is equal to unity and concentrated in the infinitesimal interval $0<x<\epsilon$ we may write

$$
-k \int_{0}^{\epsilon}\left(\frac{\partial \theta}{\partial y}\right)_{y=0} d x=1
$$

As a consequence, integration of Fq. (2.7) with respect to $x$

$$
\int_{0}^{\infty} c u \theta d y=1, \text { for } x>0 .
$$

Translated in conduction analogy this means that we must solve the conduction equation (2.6) with a unit amount of heat injected per unit area at $t=0$ into the solid. At $t=0$ the heat is concentrated near the wall and starts diffusing into the solid. During the diffusion process the total amount of heat remains constant and equal to unity. This is shown by substituting $c^{\prime}=c u$ into the integral condition (2.9) writing it in the form

$$
\int_{0}^{\infty} c^{\prime}(y) \theta d y=1
$$

which corresponds to the conduction analogy.

\section{VARIATIONAL DERIVATION OF THE TRAILING FUNCTION}

Known variational principles ${ }^{3}$ may be applied to the conduction equation (2.6). For our purpose it is convenient to transform equation (2.6) by introducing nondimensional variables. We denote by $\delta$ a characteristic thickness of the boundary layer and express the velocity profile in the form

$$
u=U \varphi(\eta)
$$

where

$$
\eta=y / \delta
$$

and $U$ represents a reference velocity. We also put

$$
\tau=\left(k x / c U \delta^{2}\right)=(1 / \mathrm{Pe})(x / \delta),
$$

where

$$
\mathrm{Pe}=e U \delta / k
$$


is a Peclet number. With these variables Eq. (2.6) becomes

$$
\varphi(\eta)(\partial \theta / \partial \tau)=\left(\partial^{2} \theta / \partial \eta^{2}\right)
$$

and the integral condition (2.9) is written

$$
\int_{0}^{\infty} \varphi(\eta) \theta d \eta=H_{0}
$$

where

$$
H_{0}=(1 / c U \delta)=(1 / k \mathrm{Pe})
$$

Equation (3.5) corresponds to thermal conduction in a semi-infinite solid of unit conductivity where $\tau$ denotes the time, $\eta$ the distance from the boundary and $\varphi(\eta)$ a heat capacity per unit volume function of $\eta$. In order to derive the trailing function we must find a solution which satisfies the adiabatic condition $\partial \theta / \partial \eta=0$ at $\eta=0$ and the integral condition (3.6) of constant total heat content.

The author's variational method is applicable to this problem. The temperature distribution is approximated by the expression

$$
\begin{array}{ll}
\theta=\theta_{0}\left[1-\left(\eta^{3} / q^{3}\right)\right], & \text { for } \quad \eta<q, \\
\theta=0, & \text { for } \eta>q ;
\end{array}
$$

where $q$ is a "penetration depth" considered to be an unknown function of time. The temperature $\theta_{0}$ at the boundary $(\eta=0)$ is a function of $q$ determined by the integral condition (3.6). This functional relation, obtained by substituting expression (3.7) for $\theta$ into the integral (3.6), is written

$$
\theta_{0}\left[A_{0}-B_{0} / q^{3}\right]=H_{0},
$$

with the following functions of $q$

$$
\begin{aligned}
& A_{0}=\int_{0}^{\alpha} \varphi(\eta) d \eta, \\
& B_{0}=\int_{0}^{\alpha} \eta^{3} \varphi(\eta) d \eta .
\end{aligned}
$$

In evaluating certain key expressions below we require the differential relationship between $\theta_{0}$ and $q$. This is readily obtained by taking total differentials of Eq. (3.8). Putting

$$
R=\frac{3 B_{0}}{A_{0} q^{3}-B_{0}}
$$

and with time derivatives $(\dot{\theta}=d \theta / d \tau$, etc) we obtain

$$
\dot{\theta}_{0} / \theta_{0}=-R(\dot{q} / q) \text {. }
$$

In the subsequent derivation $\theta_{0}$ is considered to be a given function of $q$ defined by relation (3.8). However, in order to avoid undue heaviness in the algebra, it is more convenient to postpone introducing the relationship explicitly until the last step using Eq. (3.11).

Consider first the thermal potential. In the conduction analogy corresponding to Eq. (3.5) the thermal potential is

$$
V=\frac{1}{2} \int_{0}^{q} \varphi(\eta) \theta^{2} d \eta
$$

The dissipation function is obtained by first evaluating the analog heat flow $H$ at the point $\eta$

$$
H=\int_{\eta}^{a} \theta \varphi(\eta) d \eta
$$

In order to facilitate the algebra in evaluating this expression, it is advantageous to write it in the form

$$
H=\int_{0}^{q} \theta \varphi(\eta) d \eta-\int_{0}^{\eta} \theta \varphi(\eta) d \eta .
$$

The dissipation function is

$$
D=\frac{1}{2} \int_{0}^{a} \dot{H}^{2} d \eta
$$

With the value for $\dot{H}$ obtained from (3.14) the dissipation function is given by

$$
\left(1 / \theta_{0}^{2}\right) D=\frac{1}{2} M q \dot{q}^{2}
$$

where $M$ is a function of $q$.

The variational procedure leads to the Lagrangian equation

$$
\left(1 / \theta_{0}^{2}\right)[(\partial V / \partial q)+(\partial D / \partial \dot{q})]=0 .
$$

For convenience we have divided the equation by $\theta_{0}^{2}$. The right side is zero because no heat is allowed to flow at the boundary $\eta=0$ and the corresponding thermal force $Q$ vanishes.

From expression (3.12) we derive the value

$$
\left(1 / \theta_{0}^{2}\right)(\partial V / \partial q)=-L,
$$

where $L$ is a function of $q$.

Introducing the values (3.16) and (3.18) into the Lagrangian equation (3.17) yields

$$
g(q) q \dot{q}=1
$$

where

$$
g(q)=M / L .
$$

The differential equation (3.19) is integrable by quadrature

$$
\tau=\int_{0}^{q} q g(q) d q
$$


The value of $\theta_{0}$ as a function of $q$ is obtained by writing Eq. (3.8) in the form

$$
\theta_{0}=H_{0} q^{3} /\left(A_{0} q^{3}-B_{0}\right) \text {. }
$$

Using Eqs, (3.21) and (3.22) it is possible to plot $\theta_{0}$ as a function of $\tau$ by evaluating the ordinate and abscissa as parametric functions of $q$. The resulting plot represents the trailing function and may be expressed analytically as

$$
\phi(\tau)=\theta_{0} / H_{0}=q^{3} /\left(A_{0} q^{3}-B_{0}\right)
$$

where $\phi(\tau)$ is a nondimensional form of the trailing function referred to hereafter as the reduced trailing function.

\section{VERIFICATION OF THE ACCURACY OF THE VARIATIONAL PROCEDURE}

Results obtained by the variational method outlined above may be compared with exact solutions obtainable for some simple velocity profiles.

\section{A. Plug Flow}

The simplest case

$$
\varphi(\eta)=1,
$$

corresponds to a uniform velocity distribution $u=U=$ const. Equation (3.5) becomes

$$
(\partial \theta / \partial \tau)=\left(\partial^{2} \theta / \partial \eta^{2}\right) .
$$

An exact solution of this equation satisfying the boundary condition $\partial \theta / \partial \eta=0$ at $\eta=0$ and the integral condition (3.6) is

$$
\theta_{0}=\left[H_{0} /(\pi \tau)^{\frac{1}{3}}\right] \exp \left(-\eta^{2} / 4 \tau\right) .
$$

The temperature at the wall $(\eta=0)$ is

$$
\theta_{0}=H_{0} /(\pi \tau)^{\frac{1}{2}} \text {. }
$$

For the velocity profile (4.1) the variational method with the cubic approximation (3.7) for $\theta$ yields

$$
g(q)=\frac{28}{81} .
$$

Equation (3.19) becomes

$$
q \dot{q}=\frac{81}{28} .
$$

By integration we obtain

$$
q=\left(9 / 14^{\frac{1}{2}}\right) \tau^{\frac{1}{2}} .
$$

From Eq. (3.22) we also derive

$$
\theta_{0}=\frac{4}{3}\left(H_{0} / q\right) \text {. }
$$

Elimination of $q$ between Eqs. (4.7) and (4.8) yields

$$
\theta_{0}=\left(H_{0} / 1.805\right)\left(1 / \tau^{\frac{1}{2}}\right)=0.554\left(H_{0} / \tau^{\frac{1}{2}}\right) .
$$

Comparing with the exact solution (4.4) we see that $\pi^{\frac{1}{2}}=1.772$ is replaced by the factor 1.805 , an error less than $2 \%$.

In a previous paper ${ }^{1}$ the same case was also treated by the variational method using a parabolic approximation $\theta=\theta_{0}\left(1-\eta^{2} / q^{2}\right)$ instead of the cubic expression (3.7). In this case the factor $\pi^{\frac{1}{2}}$ is replaced by 1.765 . Hence for this case the parabolic approximation is even more accurate, the error being less than a fraction of a percent. However the cubic expression (3.7) has been chosen because it provides a better approximation in the more general case.

\section{B. Linear Profile}

The case

$$
\varphi(\eta)=\eta,
$$

corresponds to a velocity profile $u=U y / \delta$ where the velocity increases linearly with the distance $y$ from the boundary.

Equation (3.5) becomes

$$
\eta(\partial \theta / \partial \tau)=\left(\partial^{2} \theta / \partial \eta^{2}\right) .
$$

An exact solution of this equation, satisfying the boundary condition $\partial \theta / \partial \eta=0$ at $\eta=0$ is

$$
\theta=C \tau^{-\frac{2}{3}} \exp \left[-\eta^{3} / 9 \tau\right]
$$

with any constant factor $C$.

This result may be verified by direct substitution into Eq. (4.11). The value of the constant is determined by substituting the solution (4.12) into the integral condition (3.6). We find

$$
C=H_{0} / \sqrt[3]{3} \Gamma\left(\frac{2}{3}\right),
$$

where $\Gamma$ represents the gamma function. The trailing function is the value $\theta_{0}$ of $\theta$ for $\eta=0$. Hence putting $\eta=0$ in Eq. (4.12) and introducing the value (4.13) of $C$ we derive

$$
\theta_{0}=0.512 H_{0} \tau^{-\frac{\pi}{3}}
$$

For comparison we now solve the same problem by the variational procedure. With $\varphi(\eta)=\eta$ we find

$$
g(q)=\frac{2}{11} q .
$$

With this value of $g(q)$, Eqs. (3.21) and (3.22) become

$$
\begin{gathered}
\tau=\frac{2}{11} \int_{0}^{a} q^{2} d q=\frac{2}{33} q^{3}, \\
\theta_{0}=\frac{10}{3} H_{0}(1 / q) .
\end{gathered}
$$

Elimination of $q$ between these two equations yields

$$
\theta_{0}=0.514 H_{0} \tau^{-\frac{2}{3}} \text {. }
$$


Comparison of this result with the exact value (4.14) shows an error less than a fraction of $1 \%$.

\section{VARIOUS WAYS OF EXPRESSING THE TRAILING FUNCTION}

The foregoing results are expressed in terms of the variables $\theta_{0}$ and $\tau$ of the nondimensional conduction analogy. The results may be expressed in equivalent form using the notation and variables of the corresponding convective problem. The notation $r(x)$ for the trailing function replaces $\theta_{0}$ and according to the definitions (3.3) and (3.6) the quantities $\tau$ and $H_{0}$ are replaced by

$$
\begin{gathered}
\tau=(1 / \mathrm{Pe})(x / \delta) \\
H_{0}=1 / k \mathrm{Pe}
\end{gathered}
$$

Consider for example the exact trailing function (4.4) for the case of uniform velocity profile. With the notation $r(x)$ instead of $\theta_{0}$, and the substitution (5.1), Eq. (4.4) is written

$$
\operatorname{Pe} k r(x)=\frac{1}{\pi^{\frac{1}{2}}}\left(\frac{1}{\operatorname{Pe}} \frac{x}{\delta}\right)^{-\frac{1}{2}} .
$$

The approximate value $(4.9)$ is written

$$
\operatorname{Pe} k r(x)=0.554\left(\frac{1}{\operatorname{Pe}} \frac{x}{\delta}\right)^{-\frac{1}{2}}
$$

Similarly for the linear velocity distribution the exact trailing function $r(x)$, corresponding to Eq. (4.14), is given in terms of $x$ and Pe by the relation

$$
\operatorname{Pe} k r(x)=\frac{1}{3^{\frac{1}{3}} \Gamma\left(\frac{2}{3}\right)}\left(\frac{1}{\mathrm{Pe}} \frac{x}{\delta}\right)^{-\frac{3}{3}} \text {. }
$$

The corresponding relation for the approximate value (4.17) is

$$
\operatorname{Pe} k r(x)=0.514\left(\frac{1}{\operatorname{Pe}} \frac{x}{\delta}\right)^{-\frac{2}{3}}
$$

In general for a boundary layer of arbitrary velocity profile we obtain a relation between $\theta_{0} / H_{0}$ and $\tau$ as given by Eq. (3.23). Such a relation may then be written in the form

$$
\operatorname{Pe} k r(x)=\phi(x / \delta \mathrm{Pe}) \text {. }
$$

The nondimensional expression $\phi$ is the reduced trailing function. This function depends only on the shape of the velocity profile.

In the two cases considered above it is also possible to express the trailing function in equivalent forms which depart from the general expression (5.6). For example consider Eq. (5.2) which corresponds to a uniform velocity profile. This case does not involve any characteristic length. Hence the parameter $\delta$ should not appear in the trailing function. This may be verified by writing Eq. (5.2) in the equivalent form

$$
k r(x)=\frac{1}{\pi^{\frac{\pi}{2}}}\left(\frac{c U x}{k}\right)^{-\frac{1}{2}}
$$

where $c U x / k$ is a Peclet number relative to the downstream abscissa $x$ instead of $\delta$.

Equation (5.4) for the linear velocity profile may also be expressed in the equivalent form

$$
k r(x)=\left[1 / 3^{\frac{1}{3}} \Gamma\left(\frac{2}{3}\right)\right](k / c)^{\frac{1}{3}}(U / \delta)^{-\frac{1}{3}} x^{-\frac{2}{3}} .
$$

This expression brings out the thermal diffusivity $k / c$ and the slope $U / \delta=d u / d y$ of the velocity profile.

\section{TRAILING FUNCTION FOR LAMINAR BOUNDARY LAYERS}

Numerical applications of the foregoing results are now given to boundary layers with laminar flow. It is assumed that the flow field has parallel stream lines. As shown below, the solution is readily extended to nonparallel streamlines without introducing any essentially new feature or difficulty. The two velocity profiles which are considered below in paragraphs $A$ and $B$ are idealized in order to analyze two extreme cases. The purpose is to show that in both cases it is possible to introduce a characteristic thickness which is defined by the same general rule valid for a whole family of velocity profiles and controls the significant features of the heat transfer.

\section{A. Piece-Wise Linear Velocity Profile}

We first consider the velocity profile represented in Fig. 1(a). The velocity increases linearly with the distance from the wall up to a thickness $\delta$ beyond which it is constant and equal to $U$. Such a distribution corresponds to the function

$$
\begin{array}{ll}
\varphi(\eta)=\eta, & \text { for } \eta<1, \\
\varphi(\eta)=1, & \text { for } \bar{\eta} \eta 1 .
\end{array}
$$

(a)

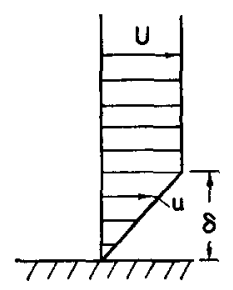

(b)

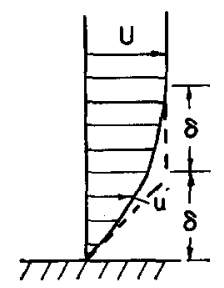

FIG. 1. Idealized velocity profiles for laminar boundary layers. (a) piece-wise linear profile; (b) parabolic profile. 


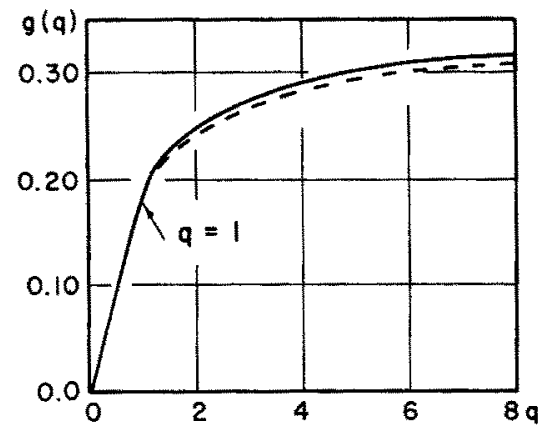

Fra. 2. Plot of $g(q)$ for the velocity profile of Fig. 1(a). The dotted line shows the value of the approximate expression $g(q)=289 /(73+819)$ for $q>1$.

We apply to this case the variational procedure with the approximation (3.7) for the temperature profile. We must distinguish two phases.

In the first phase where $q<1$ the temperature change in the fluid is restricted to the region

$$
0<y<\delta,
$$

and the problem is the same as for the linear velocity profile for which $g(q)$ is given by Eq. (4.15). In this range the reduced trailing function is obtained from Eq. (4.17) which may be written in the form (3.23)

$$
\theta_{0} / H_{0}=\phi(\tau)=0.514 \tau^{-\frac{3}{3}} \text {. }
$$

This is the value of the reduced trailing function for $q<1$ hence for $\tau<0.0606$.

In the second phase, where $q>1$, the function $g(q)$ is evaluated numerically and plotted in Fig. 2 including the straight line portion (4.15) for $q<1$. For $q=\infty$ the plot of $g(q)$ tends to an asymptotic value which as expected coincides with the constant value (4.5) obtained for the uniform velocity profile.

In the range $q>1$ the variables $\tau$ and $\theta_{0}$ are evaluated numerically as functions of $q$ using Eqs. (3.21) and (3.22). The quadrature in Eq. (3.21) is evaluated by introducing the following approximate expression in the range $q>1$ :

$$
g(q)=28 q /(73+81 q) .
$$

The approximation is represented by the dotted curve in Fig. 2. With expression (6.4) the quadrature of Eq. (3.21) is an elementary integral. We derive the numerical values of $\tau$ and $\theta / H_{0}=\phi(\tau)$ for $q>1$ and $\tau>0.0606$. They are given in Table $\mathrm{I}$ and plotted in Fig. 3.

It is possible to find a piece-wise analytical approximation for $\phi(\tau)$ which is valid for the complete range of $\tau$. We write this approximation as

$$
\theta_{0} / H_{0} \cong \phi_{1}(\tau)
$$

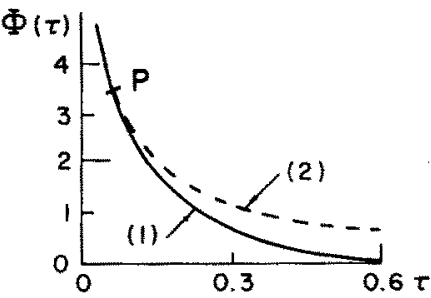

Fra. 3. Reduced trailing function $\phi(r) ;(1)$ for the turbulent boundary layer as given by equations $(7.26) ;(2)$ for the laminar boundary layer as given by values $\phi(\tau)$ in Table I. Point $P$ is the turbulent crossover point corresponding to $r=\tau_{t}=$ 0.0606 .

where

$$
\phi_{1}(\tau)= \begin{cases}0.514 \tau^{-\frac{2}{3}}, & \text { for } \quad \tau<0.64 \\ 0.554 \tau^{-\frac{1}{2}}, & \text { for } \quad \tau>0.64\end{cases}
$$

Values of $\phi_{1}(\tau)$ are shown in Table I. For $\tau<0.0606$ the value of $\phi_{1}(\tau)$ coincides with expression (6.3) derived for the first phase. However expression (6.3) remains valid as an approximation well into the second phase up to a value $\tau=0.64$. Beyond this point the approximation $\phi_{1}(\tau)$ coincides with the trailing function derived from Eq. (4.9) for a constant velocity profile.

The value $\tau=0.64$ is chosen as the crossover point because the two analytical branches of the approximation $\phi_{1}(\tau)$ are equal at that point. We may express the trailing function in the form (5.6) where $\theta_{0}$ is written as $\operatorname{Pe} k r(x)$ and $\tau=x / \delta \mathrm{Pe}$. Hence Eq. (6.5) becomes

$$
\operatorname{Pe} k r(x)=\phi_{1}(x / \delta \mathrm{Pe})
$$

In this equation the function $\phi_{1}$ is approximated by the piece-wise analytical approximation (6.6).

Some significant physical features are brought to light by considering the abscissa $x_{1}$ corresponding to the end of the first phase, where the heat starts to penetrate beyond the thickness $y=\delta$ into the region of constant velocity. The distance $x_{1}$ corresponds to $q=1$ and $\tau=0.0606$. Hence from the definition (3.3)

$$
x_{1}=0.0606 \delta \mathrm{Pe}
$$

TABLE I. Reduced trailing function $\phi(\tau)$ for the velocity profile of Fig. 1(a), and piece-wise analytical approximation $\phi_{1}(\tau)$ defined by Eq. $(6.6)$.

\begin{tabular}{llll}
\hline \multicolumn{1}{c}{$q$} & \multicolumn{1}{c}{$\tau$} & $\phi(\tau)$ & $\phi_{1}(\tau)$ \\
\hline 1.0 & 0.0606 & 3.33 & 3.33 \\
1.5 & 0.186 & 1.57 & 1.58 \\
2.0 & 0.386 & 0.993 & 0.970 \\
2.45 & 0.640 & 0.750 & 0.693 \\
3.0 & 1.02 & 0.571 & 0.548 \\
4.0 & 1.98 & 0.400 & 0.400 \\
6.0 & 4.91 & 0.250 & 0.250 \\
\hline
\end{tabular}


We may also consider the point of crossover between the two analytical approximations of Eq. (6.6) at $\tau=0.64$. This value defines a point of abscissa

$$
x_{l}=0.64 \delta \mathrm{Pe}
$$

which we refer to as the laminar crossover point. The value of $q$ at this point is $q=2.45$ and the heat has penetrated to a depth $y=2.45 \delta$ into the fluid.

It is interesting to note that the distance $x_{l}$ is about ten times the distance $x_{1}$. Furthermore in the relatively large intermediate range between $x_{1}$ and $x_{l}$ the trailing function is still represented by the same analytical approximation as for the linear velocity profile although the heat has already penetrated considerably into the region of constant velocity.

In order to provide some idea of orders of magnitude consider the case of air with

$$
\begin{aligned}
U & =10^{4} \mathrm{~cm} / \mathrm{sec}, \\
\delta & =0.06 \mathrm{~cm}, \\
k / c & =0.187 \mathrm{~cm}^{2} / \mathrm{sec}, \\
\mathrm{Pe} & =3210 .
\end{aligned}
$$

We find

$$
\begin{aligned}
& x_{1}=0.0606 \delta \mathrm{Pe}=11.7 \mathrm{~cm}, \\
& x_{\imath}=0.64 \delta \mathrm{Pe}=123 \mathrm{~cm} .
\end{aligned}
$$

\section{B. Parabolic Velocity Profile}

Consider next a boundary layer with the velocity profile shown in Fig. 1(b). The velocity profile is parabolic within a thickness $2 \delta$ beyond which the velocity is constant and equal to $U$. The function $\varphi(\eta)$ corresponding to this profile is

$$
\begin{array}{ll}
\varphi(\eta)=\eta\left(1-\frac{1}{4} \eta\right), & \text { for } \quad \eta<2, \\
\varphi(\eta)=1, & \text { for } \quad \eta>2 .
\end{array}
$$

The parameter $\delta$ in this case is the same as in the piece-wise linear profile obtained by drawing a tangent to the parabola at $y=0$. As we see the trailing function for the parabolic profile is very close to that of the piece-wise linear profile with the foregoing definition of $\delta$. Hence the length thus defined by drawing a tangent to the velocity profile at the wall may be used as a characteristic thickness and constitutes a key parameter for a whole family of velocity profiles.

The trailing function for the parabolic profile is obtained by inserting the value (6.12) for $\varphi(\eta)$ into the general equations. The procedure is entirely the same as in the preceding analysis and it is not
TaBLe II. Reduced trailing function $\phi(\tau)$ for the parabolic velocity profile of Fig. 1(b). Piece-wise analytical approximation $\phi_{1}(\tau)$ of Eq. (6.6) and improved approximation $\phi_{2}(\tau)$ of Eq. $(6.13)$.

\begin{tabular}{clrrr}
\hline & \multicolumn{1}{c}{$\tau$} & $\phi(\tau)$ & $\phi_{1}(\tau)$ & $\phi_{2}(\tau)$ \\
\hline 0.5 & 0.0069 & 14.36 & 14.40 & 14.40 \\
1.0 & 0.048 & 3.87 & 3.89 & 3.92 \\
1.5 & 0.157 & 1.87 & 1.77 & 1.85 \\
2.0 & 0.346 & 1.152 & 1.042 & 1.16 \\
2.5 & 0.616 & 0.818 & 0.710 & 0.802 \\
3.0 & 0.970 & 0.628 & 0.563 & 0.630 \\
4.0 & 1.925 & $\mathbf{0 . 4 2 8}$ & 0.399 & 0.436 \\
$\mathbf{6 . 0}$ & $\mathbf{4 . 8 5}$ & $\mathbf{0 . 2 6 0}$ & $\mathbf{0 . 2 5 2}$ & 0.267 \\
\hline
\end{tabular}

necessary to repeat the details. The values of the function $\phi(\tau)$ thus obtained are given in Table II. As announced the function $\phi(\tau)$ of Table II when plotted as a function of $\tau$ is close to the function $\phi(\tau)$ of Table I. In both cases they may be represented approximately by the piece-wise analytical expression $\phi_{1}(\tau)$ defined by Eq. (6.6). However the approximation is somewhat better for the piece-wise linear velocity profile than for the parabolic profile. The discrepancy is largest in the vicinity of the cross-over point $\tau=0.64$. The accuracy may be improved by adding a small analytical correction term. For example in the case of the parabolic profile an improved piece-wise analytical approximation is

$$
\phi_{2}(\tau)=\phi_{1}(\tau)+0.66\left[\tau /\left(1+9 \tau^{2}\right)\right] .
$$

Values of $\phi_{2}(\tau)$ are shown in Table II.

\section{TRAILING FUNCTION FOR TURBULENT BOUNDARY LAYERS}

In the case of turbulent flow with parallel streamlines the conduction analogy remains valid ${ }^{2}$ provided Eq. (2.4) is replaced by

$$
c u(\partial \theta / \partial x)=(\partial / \partial y)\left[k^{\prime}(\partial \theta / \partial y)\right]
$$

where

$$
k^{\prime}=k+c \epsilon(y) .
$$

The eddy diffusivity $\epsilon$ is a function of $y$. The coefficient $k^{\prime}$ is an effective conductivity which also depends on $y$ and represents the combined effect of molecular and eddy diffusivity.

As before we chose a reference velocity $U$ and a reference thickness $\delta$ but their definitions are different from those of the laminar case and are given below by expressions (7.9). Dimensionless variables are then introduced which are formally the same as defined by Eqs. (3.1) (3.2) and (3.3). They are $\eta=y / \delta, \quad \varphi(\eta)=u / U, \quad \tau=k x / c U \delta^{2}$. 


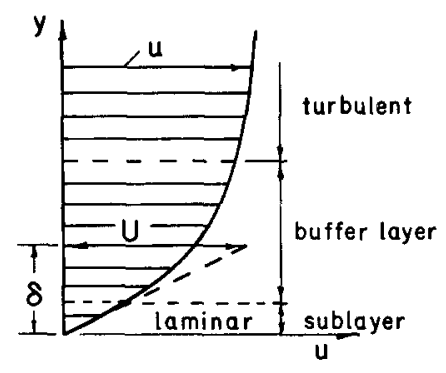

Fig. 4. Velocity profile of the turbulent boundary layer, reference thickness $\delta$ and reference velocity $U$.

With these variables Eq. (7.1) becomes

$$
\varphi(\eta)(\partial \theta / \partial \tau)=(\partial / \partial \eta)[\sigma(\eta)(\partial \theta / \partial \eta)]
$$

where

$$
\sigma(\eta)=k^{\prime} / k=1+(c \epsilon / k)
$$

By the change of variable

$$
\eta^{\prime}=\int_{0}^{\eta} \frac{d \eta}{\sigma}
$$

Eq. (7.4) is further simplified to

$$
\beta\left(\eta^{\prime}\right)(\partial \theta / \partial \tau)=\partial^{2} \theta / \partial{\eta^{\prime}}^{2}
$$

with

$$
\beta\left(\eta^{\prime}\right)=\varphi(\eta) \sigma(\eta)
$$

Equation (7.7) is now of the same form as (3.5). Again it represents a nondimensional conduction analogy and may be solved as above by applying the variational principle for conduction. However the behavior of the function $\beta\left(\eta^{\prime}\right)$ is quite different from that of $\varphi(\eta)$. Hence the solution for the trailing function will differ correspondingly from that obtained for the laminar case.

In order to derive the essential difference introduced by the presence of turbulent flow on the trailing function, consider the typical velocity profile for such a case as obtained by von Kármán ${ }^{6,7}$ and illustrated in Fig. 4.

For the purpose of the present analysis of the turbulent case it is convenient to choose for the reference thickness $\delta$ and the reference velocity $U$ the following expressions

$$
\delta=14 \nu(\rho / S)^{\frac{1}{2}}, \quad U=14(S / \rho)^{\frac{1}{2}} .
$$

In these expressions, $\nu$ is the kinematic viscosity of the fluid, $\rho$ its density and $S$ the shear stress at the wall $(y=0)$. The choice of $\delta$ is made so that the ordinate $y=\delta$ which corresponds to $\eta=1$ plays

' Th. von Kármán in Proceedings of the Fourth International Congress of Applied Mechanics, 1984 (Cambridge University Press, London, 1935) p. 54.

7 Th. von Kármán, Trans. ASME 61, 705 (1939).

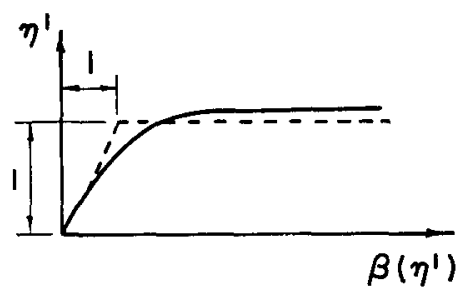

FIG. 5. Plot of $\beta\left(\eta^{\prime}\right)$. Dotted line shows piece-wise linear approximation (7.13).

a key role in the analysis. As shown in Fig. 4 the velocity defined by the second expression (7.9) is the velocity which would occur at a distance $\delta$ from the wall if the linear velocity profile of the laminar sublayer were extrapolated to that point. In the fluid mechanics literature $S$ is usually designated by $\tau_{0}$.

Three distinct regions for the velocity profile are also indicated in Fig. 4. They are

(a) the laminar sublayer for

$$
y<\frac{1}{3} \delta \text {, or } \eta<\frac{1}{3}
$$

(b) the buffer layer for

$$
\frac{1}{3} \delta<y<2 \delta, \text { or } \frac{1}{3}<\eta<2 ;
$$

(c) the fully turbulent region for

$$
y>2 \delta, \text { or } \eta>2 .
$$

The turbulent velocity profile is represented nondimensionally by the function $\varphi(\eta)$. An important feature from the standpoint of heat transfer is the behavior of the function $\sigma(\eta)$ which represents nondimensionally the combined distribution of molecular and eddy diffusivity along the velocity profile. For a Prandtl number near unity using results derived by Rannie ${ }^{8}$ it is possible to write the following approximate value:

$$
\sigma(\eta)=1+\sinh ^{2} \eta .
$$

This approximation is valid in the laminar sublayer and in the buffer layer hence for $\eta<2$. In the turbulent layer, for $\eta>2$ an approximate value is

$$
\sigma(\eta)=6.6 \eta \text {. }
$$

What is important here is the general behavior of these functions since the results derived below are not very sensitive to detailed inaccuracies in the approximation for $\sigma(\eta)$. The exact value of $\eta$ for which the two values (7.10) and (7.11) of $\sigma(\eta)$ are equal is slightly smaller than 2 .

Using a standard approximation for the velocity profile and the approximate value (7.10) of $\sigma(\eta)$ we derive the function $\beta\left(\eta^{\prime}\right)$ shown in Fig. 5. The curve

${ }^{8}$ W. D. Rannie, J. Aeron. Sci. 23, 485 (1956). 
may be approximated by the two straight lines

$$
\begin{aligned}
& \beta\left(\eta^{\prime}\right)=\eta^{\prime}, \text { for } \quad \eta^{\prime}<1 ; \\
& \beta\left(\eta^{\prime}\right)=\infty, \text { for } \quad \eta^{\prime}>1 .
\end{aligned}
$$

Since $\eta^{\prime} \cong \eta$ for $\eta^{\prime}<1$, this approximation may be replaced by

$$
\begin{aligned}
& \beta(\eta)=\eta, \text { for } \eta<1 \\
& \beta(\eta)=\infty, \text { for } \eta=1 .
\end{aligned}
$$

Hence for $\eta<1$ the differential equation (7.7) becomes

$$
\eta(\partial \theta / \partial \tau)=\partial^{2} \theta / \partial \eta^{2}
$$

It is formally the same as Eq. (4.11) for laminar flow with a linear velocity profile. The difference lies in the definition of the reference thickness $\delta$ and the reference velocity $U$ which in the case of turbulent flow are given by expressions (7.9). Another difference is represented by the approximation $\beta(1)=$ $\infty$. This is equivalent to solving Eq. (7.14) with the boundary condition

$$
\theta=0, \text { for } \eta=1 .
$$

Let us now derive the trailing function for a unit rate of heat injection concentrated at the origin $x=0$. In the nondimensional formulation this corresponds to the point $\tau=0$. The problem is solved by applying the variational principle following closely the preceding analysis for laminar flow. Again we must distinguish two phases.

In the first phase the heat penetration is restricted to the region $\eta<1$. Hence the problem is the same as for the linear velocity profile analyzed in Sec. 4 . The reduced trailing function as given by equation (4.17) is

$$
\theta_{0} / H_{0}=\phi(\tau)=0.514 \tau^{-\frac{3}{3}} .
$$

This expression is valid in the first phase for $q<1$ and $\tau<0.0606$. The end of the first phase and the beginning of the second phase corresponds to a value of $\tau$ equal to

$$
\tau_{t}=0.0606
$$

We refer to this point as the turbulent crossover point.

In the second phase $\left(\tau>\tau_{t}\right)$ the solution is of a quite different type. The temperature distribution must satisfy the boundary condition (7.15). It is obtained from expression (3.7) by substituting $q=1$. This yields

$$
\theta=\theta_{0}\left(1-\eta^{3}\right)
$$

In this expression the temperature $\theta_{0}$ is the unknown function of time. From the standpoint of the conduction analogy this second phase represents a leakage of heat from the region $\eta<1$ into an adjacent medum of infinite specific heat. With the value (7.18) for $\theta$ the thermal potential is

$$
V=\frac{1}{2} \int_{0}^{1} \eta \theta^{2} d \eta=\frac{9}{80} \theta_{0}^{2}
$$

Because of the adiabatic condition, $\dot{H}=0$ for $\eta=0$, we may write

$$
\dot{H}=-\int_{0}^{\eta} \dot{\theta} \eta d \eta=-\left(\frac{1}{2} \eta^{2}-\frac{1}{5} \eta^{5}\right) \dot{\theta}_{0} .
$$

Hence the dissipation function is

$$
D=\frac{1}{2} \int_{0}^{1} \dot{H}^{2} d \eta=\frac{63}{4400} \dot{\theta}_{0}^{2} .
$$

The dot symbol represents the derivative with respect to the variable $\tau$. The Lagrangian equation for the unknown function $\theta_{0}$ is

$$
\left(\partial V / \partial \theta_{0}\right)+\left(\partial D / \partial \dot{\theta}_{0}\right)=0 .
$$

Substitution of the expressions (7.19) and (7.21) yields the differential equation

$$
\theta_{0}+\frac{7}{55} \dot{\theta}_{0}=0 .
$$

The constant of integration is determined by the value of $\theta_{0}$ at the end of the first phase. This value of $\theta_{0}$ is obtained by putting $\tau=\tau_{t}=0.0606$ in expression (7.16). We obtain

$$
\theta_{0}=3.33 H_{0} .
$$

The corresponding solution of Eq. (7.23) is

$\theta_{0} / H_{0}=\phi(\tau)=3.33 \exp \left[-\frac{55}{7}\left(\tau-\tau_{i}\right)\right]$.

This is the value of the reduced trailing function in the second phase.

From Eqs. (7.16) and (7.25) we conclude that the reduced trailing function is represented approximately by the piece-wise analytical expression

$\phi(\tau)= \begin{cases}0.514 \tau^{-\frac{2}{3}}, & \text { for } \tau<\tau_{t}, \\ 3.33 \exp \left[-\frac{55}{7}\left(\tau-\tau_{t}\right)\right], & \text { for } \tau>\tau_{t},\end{cases}$

where $\tau_{t}$ is the value (7.17) corresponding to the turbulent crossover point. The reduced trailing function (7.26) is plotted in Fig. 3. In contrast with the trailing function for laminar flow it decays much faster with the distance from the point of injection. In fact beyond a point corresponding to $\tau=0.6$, it may be considered as having vanished and we may put $\phi(\tau)=0$. 


\section{A. Possibility of Large Errors in Thermal Stress Analysis Based on Local Heat Transfer Coefficient}

Referring to the numerical values (6.10) and (6.11) we conclude that in both laminar and turbulent flow, local heat injection exerts an influence downstream up to distances which may be of the order of $10^{2} \mathrm{~cm}$. This points to large possible errors which may result from using a local heat transfer coefficient in transient thermal stress analysis.

There seems to be a current misunderstanding as to what is involved here. The fact that the local heat transfer depends on upstream temperatures of the fluid-solid interface is well known as shown by the integral relation derived by Leveque ${ }^{4}$ as early as 1928 and many similar analyses by later authors. However, it was generally assumed that for streamwise temperature distributions encountered in practice it was possible to use an "equivalent" local heat transfer coefficient derived by an averaging process. That this may not be adequate even for a smooth distribution was shown earlier by the author on a simple example. ${ }^{2}$ The present results provide further quantitative confirmation of this conclusion in a wide range of problem for laminar and turbulent flow. It also follows that the use of a local heat transfer coefficient suggests an erroneous physical model even as an approximation for a smooth temperature distribution. The correct physical model derived earlier ${ }^{2}$ and in the present analysis provides an explanation of some unorthodox behavior along with a simple quantitative evaluation.

Furthermore, in transient thermal stress analysis, we do not know in advance the streamwise temperature distribution. Use of a local heat transfer coefficient obtained by an a priori estimate of the temperature distribution and a weighted average of the upstream temperature may lead to significant errors. This is partieularly true for thermal stresses because they are very sensitive to temperature differences.

\section{B. Application of the Trailing Function to Ducted Flows}

In the foregoing analysis we have assumed parallel streamlines. This assumption is rigorously valid for the case of flow in a straight duct with a fully developed velocity profile. The variational procedure outlined above is readily applicable and provides a correct evaluation of the trailing function for ducted flow. Because of the confined nature of the flow the reduced trailing function $\phi(\tau)$ instead of vanishing for $\tau=\infty$ will tend toward an asymptotic value greater than zero. This asymptotic value is immediately obtained without additional calculation and is equal to $\theta=1 / c w$ where $w$ is the total volume flow per second in the duct and $c$ is the heat capacity per unit volume.

For boundary layer heat transfer in a nonconfined fluid or for ducted flow under entrance conditions the assumption of parallel streamlines may not be valid. However as shown in the following section the foregoing results are easily extended to this case.

\section{GENERALIZED CONDUCTION ANALOGY FOR NONPARALLEL STREAMLINES}

The conduction analogy may be generalized to convective heat transfer in a flow field with nonparallel streamlines as already pointed out ${ }^{2}$ by applying the von Mises transformation. However application of the variational method to the resulting equation is not convenient because of the presence of some artificial singular features introduced by the von Mises transformation. Therefore we introduce a transformation which is more general and leads to a more convenient formulation.

As before we assume the fluid to be incompressible. The components of the two-dimensional velocity field are $u(x y)$ and $v(x y)$. The excess temperature $\theta$ of the fluid above the adiabatic value satisfies the approximate equation

$$
c u(\partial \theta / \partial x)+c v(\partial \theta / \partial y)=(\partial / \partial y)\left[k^{\prime}(\partial \theta / \partial y)\right]
$$

which is the same as Eq. (7.1) with the addition of the convective term $c v \partial \theta / \partial y$ in the $y$ direction. The equation is valid for either laminar or turbulent flow and the total conductivity $k^{\prime}$ is given by expression (7.2). Consider a change of variables

$$
\begin{aligned}
& y^{\prime}=y^{\prime}(x y), \\
& x^{\prime}=x^{\prime}(x y) .
\end{aligned}
$$

These variables may be interpreted as curvilinear coordinates. We choose $y^{\prime}$ such that the coordinate lines

$$
y^{\prime}=\text { const }
$$

coincide with the streamlines. Hence

$$
u \frac{\partial y^{\prime}}{\partial x}+v \frac{\partial y^{\prime}}{\partial y}=0
$$

We may write

$$
\begin{aligned}
& \frac{\partial \theta}{\partial x}=\frac{\partial \theta}{\partial x^{\prime}} \frac{\partial x^{\prime}}{\partial x}+\frac{\partial \theta}{\partial y^{\prime}} \frac{\partial y^{\prime}}{\partial x} \\
& \frac{\partial \theta}{\partial y}=\frac{\partial \theta}{\partial x^{\prime}} \frac{\partial x^{\prime}}{\partial y}+\frac{\partial \theta}{\partial y^{\prime}} \frac{\partial y^{\prime}}{\partial y}
\end{aligned}
$$

We now choose a particular case of the transforma- 
tion (8.2) by putting $x^{\prime}=x$. Taking into account relations (8.4) and (8.5) we conclude

$$
\begin{gathered}
u \frac{\partial \theta}{\partial x}+v \frac{\partial \theta}{\partial y}=u \frac{\partial \theta}{\partial x^{\prime}}, \\
\frac{\partial \theta}{\partial y}=\frac{\partial \theta}{\partial y^{\prime}} \frac{\partial y^{\prime}}{\partial y} .
\end{gathered}
$$

Using these results leads to a simplified form of Eq. (8.1). Further simplification is obtained by putting

$$
\partial y^{\prime} / \partial y=1 / \alpha\left(x^{\prime} y^{\prime}\right)
$$

and writing $x$ instead of $x^{\prime}$ since this is only a change of notation. Equation (8.1) becomes

$$
\operatorname{cu\alpha }(\partial \theta / \partial x)=\left(\partial / \partial y^{\prime}\right)\left[\left(k^{\prime} / \alpha\right)\left(\partial \theta / \partial y^{\prime}\right)\right] .
$$

Consider two streamlines of coordinates $y_{1}^{\prime} y_{2}^{\prime}$. The integral

$$
\int_{\nu_{\mathrm{t}}}^{y_{2}} u d y=\int_{y_{\mathrm{t}^{\prime}}}^{u_{2_{2}^{\prime}}} u \frac{\partial y}{\partial y^{\prime}} d y^{\prime}=\int_{\nu_{\mathrm{t}^{\prime}}}^{u_{2}{ }^{\prime}} u \alpha d y^{\prime}
$$

represents the volume flow between the two streamlines. Since the fluid is incompressible this quantity is independent of $x$. Hence $u \alpha$ is independent of $x$ and we may write

$$
u \alpha=u_{r}\left(y^{\prime}\right) .
$$

Let us choose the streamline coordinates in such a way that $y^{\prime}=y$ at a certain reference abscissa $x_{r}$. In that case $u_{r}\left(y^{\prime}\right)$ is the velocity profile at the reference abscissa. Equation (8.8) becomes

$$
c u_{r}\left(y^{\prime}\right)(\partial \theta / \partial x)=\left(\partial / \partial y^{\prime}\right)\left[\left(k^{\prime} / \alpha\right)\left(\partial \theta / \partial y^{\prime}\right)\right] .
$$

We note that $k^{\prime} / \alpha$ is a function of $x$ and $y^{\prime}$. Hence Eq. (8.11) represents a one-dimensional conduction analogy where $x$ plays the role of a time variable and $y^{\prime}$ is a space coordinate. The author has shown ${ }^{3}$ that the variational principle and the corresponding Lagrangian equations are applicable to thermal conduction with a specific heat which depends on the coordinates and a thermal conductivity which depends on both the time and the coordinates. As a consequence, Eq. (8.11), which governs convective laminar and turbulent heat transfer with nonparallel streamlines, may be solved by variational methods.

In particular the method may be applied to the evaluation of the trailing function for boundary layers with nonparallel streamlines. Let the origin of $x$ be located at the point of injection. We also choose the origin as the reference abscissa $x_{r}$. In that case $u_{r}\left(y^{\prime}\right)$ is the velocity profile at the point of injection and $x$ represents the distance downstream from this point. For this local velocity profile $u_{r}\left(y^{\prime}\right)$, it is possible to define a reference velocity $U$ and a reference thickness $\delta$ which have been derived in the preceding analysis for either laminar or turbulent flow and will depend on the location of the point of injection. With nondimensional variables we write

$$
u_{r} / U=\varphi(\eta), \quad \eta=y^{\prime} / \delta .
$$

Consider now the behavior of the function $k^{\prime} / \alpha c$ downstream from the origin. It is a function of $x$ and $y^{\prime}$. Since the origin is the reference abscissa for which $\alpha=1$ the value of $k^{\prime} / \alpha c$ on the $y$ axis is

$$
k^{\prime} / \alpha c=(1 / c) k^{\prime}\left(y^{\prime}\right)
$$

In this expression $(1 / c) k^{\prime}\left(y^{\prime}\right)=1 / c k^{\prime}(y)$ is the total diffusivity along the $y$ axis. The value of $k^{\prime} / \alpha c$ is also a function of the downstream distance $x$ from the point of injection. In most cases this function may be represented approximately as

$$
\left(k^{\prime} / \alpha c\right)=(1 / c) k^{\prime}\left(y^{\prime}\right) f(x)
$$

with the property $f(0)=1$. This means that the distribution of $k^{\prime} / \alpha c$ varies only by a change of scale as we move downstream. Provided there is no sudden transition this approximation is justified if we remember that the trailing function tends to vanish at a certain distance downstream so that the approximation need be valid only in the downstream neighborhood of the point of injection. Note also that the function depends on the location of the point of injection. We now put

$$
\begin{gathered}
\sigma(\eta)=\frac{1}{k} k^{\prime}\left(y^{\prime}\right), \\
\tau=\frac{k}{U_{c} \delta^{2}} \int_{0}^{x} f(\lambda) d \lambda .
\end{gathered}
$$

With these variables Eq. (8.11) becomes

$$
\varphi(\eta)(\partial \theta / \partial \tau)=(\partial / \partial \eta)[\sigma(\eta)(\partial \theta / \partial \eta)] .
$$

This result coincides with Eq. (7.4). As a consequence the trailing function $\phi(\tau)$ derived previously is directly applicable to the case of nonparallel streamlines provided the definitions (8.15) are introduced for $\tau$ and $\sigma$. We may use the piece-wise analytical approximations (6.6) or (7.26) depending on the laminar or turbulent nature of the flow. The difference with the case of parallel streamlines lies in the definitions (8.15) of the variables $\tau$ and $\sigma$ in addition to the fact that $U, \delta$ and $f(x)$ depend on the location of the point of injection. The function $f(x)$ may be approximated in various ways. For example we may use the expression

$$
f(x)=1-\mu x,
$$


where $\mu$ is a coefficient depending on the point of injection. In this case

$$
\tau=\left(k x / U_{c} \delta^{2}\right)\left(1-\frac{1}{2} \mu x\right) .
$$

Other more flexible but less simple approximations may be used. A possible expression is

$$
f(x)=(1-\mu x)^{n}
$$

where $n$ is a fractional exponent depending also on the location of the point of injection.

\section{TYPICAL APPLICATIONS OF THE TRAILING FUNCTION}

The trailing function is a central concept which embodies the essentials of the physics of heat transfer from solid to fluid. It was introduced specifically by the author ${ }^{1}$ for the purpose of solving the coupled problem of transient heat conduction in a solid adjacent to a moving fluid using the variational Lagrangian approach. This provides a correct and relatively simple formulation of an otherwise very complex problem without having to introduce the erroneous concept of local heat transfer coefficient.

At the same time the trailing function also provides immediate solutions for the usual simpler problems with prescribed wall temperature or heat injection, and those of heat transfer between moving fluids in heat exchangers.

\section{A. Heat Transfer with Prescribed Wall Conditions}

Consider the problem of evaluating the wall temperature for a prescribed distribution of heat injection into the fluid. An immediate solution of this problem follows from the definition itself of the trailing function. In order to illustrate this let the point of heat injection be located at the abscissa $x=\xi$. At this point a unit amount of heat is injected per unit time. The distance downstream from the point of injection is now $x-\xi$. Hence we must replace $x$ by $x-\xi$ in expression (8.15) for $\tau$. It becomes

$$
\tau(x-\xi, \xi)=\frac{1}{\operatorname{Pe}(\xi) \delta(\xi)} \int_{0}^{x-\xi} f(\xi, \lambda) d \lambda,
$$

where

$$
\operatorname{Pe}(\xi)=(c / k) \delta(\xi) U(\xi) .
$$

The thickness $\delta(\xi)$ the velocity $U(\xi)$ and the corresponding Peclet number $\mathrm{Pe}(\xi)$ are local values depending on the abscissa $\xi$ of the point of injection. The function $f(\xi, \lambda)$ also depends on the particular nature of the flow field downstream from the point of injection.
For the particular case of parallel streamlines where $\delta$ and $U$ are constant and $f(\xi, \lambda)=1$ expression (9.1) reduces to

$$
\tau=(x-\xi) / \delta \mathrm{Pe},
$$

which is the same as (3.3) after replacing $x$ by $x-\xi$.

Equation (3.23) is valid for the case of nonparallel streamlines. Hence

$$
\theta_{0}=H_{0} \phi(\tau) .
$$

In the reduced trailing function $\phi(\tau)$ the argument $\tau$ is defined by Eq. (9.1) while $H_{0}$ is given by

$$
H_{0}=1 / k \operatorname{Pe}(\xi) .
$$

This expression is derived from the definition (3.6) using the local value (9.2) of the Peclet number at the point of injection. From relations (9.4) and (9.5) we conclude that the trailing function is

$$
\theta_{0}=r(x-\xi, \xi)=[1 / k \operatorname{Pe}(\xi)] \phi(\tau) .
$$

The function $r(x-\xi$, $\xi$ represents the wall temperature rise at point $x$ for a unit rate of heat injection at point $\xi$. We note the property

or

$$
\begin{aligned}
& \phi(\tau)=0, \text { for } \quad \tau<0, \\
& \phi(\tau)=0, \text { for } x<\xi,
\end{aligned}
$$

which expresses the fact that there is no temperature rise upstream from the point of injection.

Consider now a continuous distribution of heat injection equal to $\dot{H}(\xi)$ per unit area at a point $\xi$. Assume that the injection is applied only in the region downstream from the abscissa $x_{0}$. At any point $x>x_{0}$ downstream there is a temperature rise $\theta(x)-\theta_{a}(x)$ which depends on the total heat injection between $\xi=x_{0}$ and $\xi=x$. Hence

$$
\theta(x)-\theta_{a}(x)=\int_{x_{0}}^{x} r(x-\xi, \xi) \dot{H}(\xi) d \xi .
$$

By introducing expression (9.6) we derive

$$
\theta(x)-\theta_{a}(x)=\frac{1}{k} \int_{x_{0}}^{x} \frac{\phi(\tau)}{k \operatorname{Pe}(\xi)} \dot{H}(\xi) d \xi .
$$

This yields the wall temperature $\theta(x)$ directly as a quadrature for any given distribution $\dot{H}(\xi)$ of heat injection into the fluid at the wall. The adiabatic temperature $\theta_{a}(x)$ is the wall temperature in the absence of heat injection.

Relation (9.9) also provides an integral equation for the inverse problem of determining the distribution $\dot{H}(\xi)$ of heat transfer where the temperature $\theta(x)$ is given at the wall. In the general case this integral equation may conveniently be solved 
numerically. A standard procedure is to replace the integral equation by a system of linear algebraic equations with the values of $\dot{H}(\xi)$ at a finite number of discrete stations as unknowns. Linear or parabolic interpolation will improve the accuracy of the quadratures and take care of the formal difficulty due to the singularity of $\phi(\tau)$ at $\tau=0$. Another method is to replace the distribution $\dot{H}(\xi)$ by a sum of known functions with unknown coefficients. In particular we may use a polynomial with its coefficients as unknown.

\section{B. Heat Transfer in Heat Exchangers}

Two fluids flowing in opposite direction are separated by a thin solid wall. The heat transfer between the two fluids is assumed to take place between the abscissas $x_{0}$ and $x_{1}$. The first fluid flows in the $x$ direction. Its trailing function is $r(x-\xi, \xi)$ and its adiabatic temperature is $\theta_{a}(x)$. The wall temperature for the first fluid is

$$
\theta(x)=\theta_{a}(x)+\int_{x_{0}}^{x} r(x-\xi, \xi) \dot{H}(\xi) d \xi
$$

where $\dot{H}(\xi)$ is the local rate of heat transfer from the second to the first fluid.

The second fluid flows in the negative direction of $x$. Its trailing function is $r^{\prime}(\xi-x, \xi)$ and its adiabatic temperature $\theta_{a}^{\prime}(x)$. The wall temperature of the second fluid is

$$
\theta^{\prime}(x)=\theta_{a}^{\prime}(x)-\int_{x}^{x_{1}} r^{\prime}(\xi-x, \xi) \dot{H}(\xi) d \xi .
$$

The temperature difference across the separating wall is

$$
\theta^{\prime}(x)-\theta(x)=\left[a / k_{s}\right) \dot{H}(x),
$$

where $a$ is the thickness of the separating wall and $k_{s}$ its thermal conductivity. By combining relations (9.10), (9.11), and (9.12), we derive

$$
\begin{aligned}
\theta_{a}^{\prime}(x)- & \theta_{a}(x)=\int_{x}^{x_{1}} r^{\prime}(\xi-x, \xi) \dot{H}(\xi) d \xi \\
& +\int_{x_{0}}^{x} r(x-\xi, \xi) \dot{H}(\xi) d \xi+\frac{a}{k_{s}} \dot{H}(x) .
\end{aligned}
$$

This is an integral equation for the unknown heat transfer distribution. It is readily solved numerically by the standard procedures already mentioned above for the solution of Eq. (9.9). In problems of heat exchangers the unknown $\dot{H}(x)$ may conveniently be approximated by a sum of a small number of exponential distribution of unknown amplitude. In most cases the adiabatic temperature difference $\theta^{\prime}-\theta$ will be independent of $x$.

\section{Coupled Transient Heat Conduction and Boundary- Layer Heat Transfer}

The foregoing examples refer to the solution of simpler problems of boundary-layer heat transfer. A more important application of the trailing function is to the transient conduction problem in a solid adjacent to a moving fluid. The method has been developed in detail in an earlier paper ${ }^{1}$ for the most general three-dimensional case. We briefly recall the method in the more restricted context of the present paper assuming the problem to be twodimensional in the $x y$ plane.

The thermal vector field $H_{i}$ in the solid is defined in terms of generalized coordinates $q_{i}$ as

$$
H_{i}=H_{i}\left(q_{1} q_{2} \cdots q_{n} x y t\right) .
$$

The generalized coordinates $q_{i}$ satisfy the Lagrangian type differential equations

$$
\left(\partial V / \partial q_{i}\right)+\left(\partial D / \partial \dot{q}_{i}\right)+C_{i}=Q_{i}
$$

where $V$ and $D$ are, respectively, the thermal potential and the dissipation function in the solid. The term $Q_{i}$ is the thermal force associated with the adiabatic temperature at the boundary adjacent to the fluid. If we denote by $s$ downstream distance measured along the curved boundary, we write

$$
Q_{i}=-\int_{s} \theta_{a}(s) \frac{\partial H_{n}(s)}{\partial q_{i}} d s .
$$

The outward normal component of $H_{i}$ at point $s$ of the boundary is denoted by $H_{n}(s)$.

The terms $C_{i}$ represent the heat transfer properties of solid to fluid and is written

$C_{i}=\int_{s} \int_{s^{\prime}} \frac{\partial H_{n}(s)}{\partial q_{i}} \dot{H}_{n}\left(s^{\prime}\right) r\left(s-s^{\prime}, s^{\prime}\right) d s d s^{\prime}$.

By definition $\dot{H}_{n}$ is the total time derivative of $H_{n}$ hence

$$
\dot{H}_{n}=\sum \frac{\partial H_{n}}{\partial q_{i}} \dot{q}_{i}+\frac{\partial H_{n}}{\partial t} .
$$

In general, if the curvature of the boundary is not too large, the trailing function $r\left(s-s^{\prime}, s^{\prime}\right)$ may be assumed to be the same as $r(x-\xi, \xi)$ for the straight boundary, putting $x=s$ and $\xi=s^{\prime}$.

The transient heat conduction in the solid is then determined by solving the Lagrangian differential equations (9.15) for the time dependent unknowns.

\section{ACKNOWLEDGMENTS}

The author is indebted to Dr. A. Winzer for assistance in the analytical and numerical work.

This work was supported by the Air Force Office of Scientific Research of the Office of Aerospace Research under Contract No. AF49(638)-1329. 